\title{
Lactic Acid Is an Independent Predictor of Mortality and Improves the Predictive Value of Existing Risk Scores in Patients Presenting With Acute Gastrointestinal Bleeding
}

\author{
Matthew Berger ${ }^{\mathrm{a}}$, Vadim Divilov ${ }^{\mathrm{a}}$, Getu Teressa ${ }^{\mathrm{a}, \mathrm{b}}$
}

\begin{abstract}
Background: There are validated clinical risk scores for risk stratifying patients presenting with acute upper gastrointestinal bleed (GIB), including Glasgow-Blatchford score (GBS), Pre-endoscopic Rockall score (RS-PE) and post-endoscopic complete Rockall Score (RS-C), and AIMS65. Several studies have explored the predictive value of lactic acid (LA) in the context of GI bleeding, but the prognostic role of LA and its incremental value in combination with existing clinical risk scores is not well defined.
\end{abstract}

Methods: We conducted a retrospective analysis of consecutive patients presenting to the emergency department of a single large academic tertiary care center from January 2014 to December 2015 with a charted diagnosis of acute GIB, inclusive of both upper and lower sources. We evaluated the independent role of LA as well as three clinical risk scores for predicting in-hospital mortality in these patients.

Results: Out of 704 patients admitted with acute GI bleeding, 366 patients had LA measured on presentation to the emergency department. The mean LA level, GBS, RS-PE and RS-C were found to be significantly higher in non-survivors, while there was no difference in the mean AIMS65 score between survivors and non-survivors. A multivariate logistic regression analysis showed that LA level was an independent predictor of in-hospital mortality. The area under the curve (AUC) for the receiver operator characteristic for RS-C, RS$\mathrm{PE}$, and GBS were $0.742,0.675$, and 0.652 , respectively. When integrating LA into the above risk scores, the AUC for RS-C, RS-PE, and GBS showed statistical significance improvements to $0.780(\mathrm{P}=$ $0.04), 0.774(\mathrm{P}=0.012)$, and $0.706(\mathrm{P}=003)$, respectively.

Conclusions: In unselected patients with GIB who presented to the emergency department, LA is an independent predictor of in-hospital mortality. Integration of LA into RS-C, RS-PE, and GBS risk scores improved their ability to predict in-hospital mortality. The modified

Manuscript submitted September 5, 2018, accepted September 19, 2018

aDepartment of Internal Medicine, Stony Brook Medicine, Stony Brook, NY, USA

bCorresponding Author: Getu Teressa, Department of Internal Medicine, Health Sciences Tower, Level 16, Room 020, Stony Brook, NY 11794-8160, USA. Email: getu.teressa@stonybrookmedicine.edu

doi: https://doi.org/10.14740/gr1085w
LA-based RS-PE (L-Rockall pre-endoscopic) score demonstrated predictive value comparable to the post-endoscopic RS-C.

Keywords: Risk scores; Gastrointestinal bleeding; Mortality; Lactic acid

\section{Introduction}

The annual incidence of hospitalization for acute gastrointestinal bleed (GIB) is 36 per 100,000 population for acute lower GIB and about half of that for upper GIB [1]. Upper GIB has been estimated to account for up to 20,000 deaths annually in the United States [2], while mortality rates ranging between $2 \%$ to $10 \%$ have been reported for lower GIB [3]. Appropriate risk stratification of patients presenting with acute GIB aids in the triage of patients to determine need for hospital admission and level of in-hospital care, as well as the need for emergent endoscopic intervention.

Several well-validated risk stratification tools have been developed specifically for upper GI bleeds, the most commonly used being the Glasgow-Blatchford score (GBS), Rockall Score, and AIM65 score. Unlike risk scores for upper GIB, there are no risk scores with high generalizability that have been established for acute lower GIB.

Although the use of risk stratification systems are strongly encouraged in current practice guidelines, only $53 \%$ of physicians had ever heard of and 30\% had ever used an upper GIB risk scores in a nationwide study in the United States [4]. The possible barriers to adherence are not clearly identified. Since separate risk scores are derived for upper and lower GIB, their application in clinical practice dictates accurate clinical determination of the site of GIB, which is not always clear during early evaluation by frontline providers. For instance, approximately $15 \%$ of patients with presumed lower GIB are ultimately found to have an upper GIB source for their bleeding [5], underscoring uncertainty in discerning upper vs. lower source of GIB, and therefore limiting the utility of scores specifically developed for upper or lower GIB. There are no risk scores that have been validated for or evaluated in a mixed population of upper and lower GIB.

Existing risk scores already incorporate a variety of laboratory parameters into their formulae: GBS uses Blood Urea 
Nitrogen (BUN) \& Hemoglobin, and AIMS65 uses Albumin \& International Normalized Ratio (INR). However, multiple recent studies have demonstrated the significant role Lactic Acid (LA) plays as a predictor of clinical outcomes, demonstrated in upper GIB patients [6-11] and mixed upper \& lower GIB patients $[12,13]$. In a retrospective study of 331 patients with upper GIB, LA was shown to enhance the performance of two scoring systems (GBS and the post-endoscopic Rockall score) in predicting mortality, although the improvement in prognositic performance might not to be clinically significant [10].

The aim of this study was twofold: 1) To validate existing risk scores (GBS, AIMS65, and RS) to predict in-hospital mortality in unselected patients presenting with acute GIB, suspected of either upper or lower GI bleeds; 2) To evaluate the role of initial venous LA to independently predict mortality and its incremental value to the above clinical risk scores.

\section{Materials and Methods}

\section{Study design and patient selection}

We conducted a retrospective analysis of consecutive patients presenting to the emergency department (ED) of a single large academic tertiary care medical center from January 2014 to December 2015 with a charted diagnosis of acute GIB, inclusive of both upper and lower sources. Gastroenterologists and general surgeons are available $24 \mathrm{~h}$ daily, 7 days a week for emergent endoscopy or surgery.

The medical records were queried for patients' demographic characteristics, medical history, initial vital signs, laboratory values, and the primary outcome of in-patient mortality status. Chart review of all physician notes written within the first 12 $\mathrm{h}$ of presentation was used to determine symptoms of melena, altered mental status and syncope. Endoscopic diagnosis and hemorrhagic stigmata were determined by review of all endoscopic reports. Glasgow-Blatchford Bleeding Score (GBS), AIMS65, pre-endoscopic Rockall (RS-PE) and post-endoscopic Rockall score (RS-C) were calculated for each patients. RS-C was calculated only for the 189 patients who had endoscope.

\section{Statistics}

Continuous variables were reported as mean \pm standard deviation or median \pm interquartile range (IQR) and compared by $t$ test for parametric variables or Mann-Whitney U test for nonparametric variables. Categorical variables were presented as counts (percentages) and compared by using either Chi-square test or Fisher's exact test (for sparse data). A P-value of $<0.05$ was considered statistically significant. Variables found to be statistically significant in univariate analysis were included as candidate covariates in the multivariate logistic regression analysis which was run using forward stepwise selection procedure.

To evaluate the incremental value of LA to clinical risk scores, we generated modified scores (L-GBS, L-RS-PE, and L-RS-C) by adding extra points to the calculated scores if LA level was elevated above the optimal threshold. The optimal cut-off for LA for discriminating in-patient mortality was a value that corresponds to the Youden's-index (maximum value of sensitivity + specificity - 1). The numerical value of the extra point to be added to the risk scores was obtained from the beta coefficient $(95 \%$ confidence interval (CI)) of a binary logistic regression model in which the variable "lactic acid" was dichotomized into a value above or below the optimal cut-off determined by the Youden's Index. The discriminative ability of the original and modified risk scores for predicting in-hospital mortality was evaluated by calculating the AUC of the receiver operating characteristic (ROC) curves and which were compared using nested logistic regression models.

STATA 14 (StataCorp LLC, College Station, Texas) was used to plot the ROC curves and perform nested logistic regression models to assess the improvement in AUC after incorporating LA in risk models. The remaining analyses were performed with IBM ${ }^{\circledR}$ SPSS Version 24.

\section{Ethics statement}

The Stony Brook University Hospital Institutional Review Board (IRB) approved this retrospective cohort study. The requirement for written informed consent from patients was waived.

\section{Results}

\section{Population characteristics}

Out of 704 patients admitted with acute GIB, 366 patients had venous LA measured within $24 \mathrm{~h}$ of hospital admission, of whom 30 died during their index hospitalization. The baseline demographic and clinical characteristics of these patients according to in-patient mortality status are shown in Table 1. Compared to survivors, patients who died in the hospital were older (mean age: 70.5 vs. 76.6 years, $\mathrm{P}=0.021$ ), had higher LA level (mean 2.2 vs. $5.8 \mathrm{mmol} / \mathrm{L}, \mathrm{P}<0.0001$ ), more likely to have albumin levels $<3 \mathrm{~g} / \mathrm{dL}(16.4 \%$ vs. $50 \%, \mathrm{P}<0.0001)$, had lower mean systolic blood pressure (127 vs. $116 \mathrm{~mm} \mathrm{Hg}$, $\mathrm{P}=0.035)$, and were more likely to present with altered mental status $(7.1 \%$ vs. $23.3 \%, \mathrm{P}=0.008)$ and syncope $(5.7 \%$ vs. $16.7 \%, \mathrm{P}=0.037)$. The mean BUN trended higher in non-survivors (33.3 vs. $41.5 \mathrm{mg} / \mathrm{dL}, \mathrm{P}=0.058$ ).

Furthermore, as shown in Table 2, non-survivors had statistically significant higher mean GBS ( 8 vs. $10.7, \mathrm{P}=0.002$ ), RS-PE (3.6 vs. $4.6, \mathrm{P}=0.001)$, and RS-C (5.3 vs. $6.9, \mathrm{P}=$ $0.004)$ scores while there was no difference in AIMS65 score between survivors and non-survivors ( 1.87 vs. $1.90, \mathrm{P}=0.683$ ).

In a stepwise logistic regression model that incorporated variables that were significant in the univariate analysis (Table $3)$, LA remained an independent predictor of in-hospital mortality (odds ratio (OR): 1.32 (95\% CI: $1.11-1.57, \mathrm{P}<0.0001)$ ), as did age (OR: 1.07 (95\% CI: $1.02-1.11, \mathrm{P}<0.0001)$ ), and albumin level (OR: 0.28 (95\% CI: $0.12-0.64, \mathrm{P}<0.0001)$ ).

The optimal LA cut-off $(2.75 \mathrm{mmol} / \mathrm{L})$ was determined by calculating the Youden's Index. 
Table 1. Baseline Demographic Characteristics and Test Result of Patients With Acute Gastrointestinal Bleeding According to InPatient Mortality Status

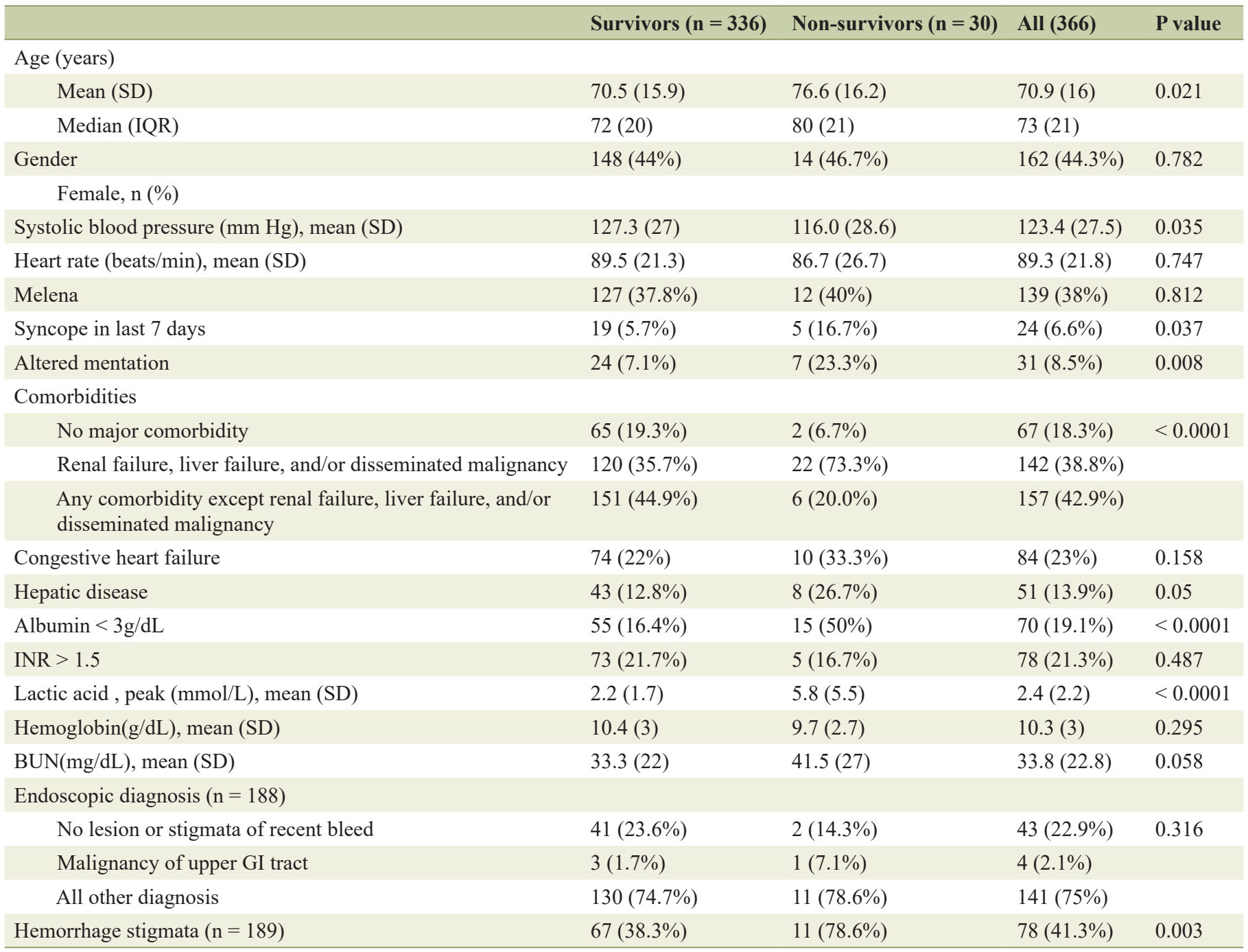

To estimate the weighted score (point) of LA in mortality prediction in the modified risk scores, we used values within the $95 \%$ CI of the beta coefficient obtained from a logistic regression model that used the same variables shown in Table 3, except the LA level was dichotomized at the optimal cut-off value $(<$ or $\geq 2.75 \mathrm{mmol} / \mathrm{L}$ ) (Supplementary Table 1 , www. gastrores.org).

Based on the calculated beta coefficient of 2.32 (95\% CI:
1.3 - 3.3), we added 1, 2, or 3 points to each patient's calculated risk scores if LA level is $\geq 2.75 \mathrm{mmol} / \mathrm{L}$, thereby deriving L1-, L2-, and L3- modified risk scores. Since there was no difference in AIMS65 score between survivors and non-survivors, modified AIMS65 score was not generated.

The power of the clinical risk scores for discriminating inpatient mortality was evaluated by ROC plots and the AUC of each ROC curves (Fig. 1, Table 4). The AUC of the origi-

Table 2. Clinical Risk Scores According to Inpatient Mortality Status

\begin{tabular}{llll} 
Risk scores & Survivors $(\mathbf{n}=\mathbf{3 3 6})$ & Non-survivors $(\mathbf{n}=\mathbf{3 0})$ & P value \\
\hline Blatchford score & $8(4.6)$ & $10.7(4.3)$ & 0.002 \\
Rockall-pre-endoscopy & $3.6(1.5)$ & $4.6(1.3)$ & 0.001 \\
Rockall-complete* & $5.3(2.1)$ & $6.9(1.3)$ & 0.004 \\
AIMS65 & $1.87(0.8)$ & $1.9(0.8)$ & 0.683 \\
\hline
\end{tabular}

*Values are mean score (SD). 
Table 3. Binary Logistic Regression for the Prediction of In-Patient Mortality in Patients With Acute Gastrointestinal Bleeding

\begin{tabular}{|c|c|c|c|c|}
\hline & \multirow{2}{*}{ Odds ratio (OR) } & \multicolumn{2}{|c|}{ 95\% Confidence interval for OR } & \multirow{2}{*}{ P value } \\
\hline & & Lower & Upper & \\
\hline Age & 1.07 & 1.02 & 1.11 & $<0.0001$ \\
\hline Hepatic disease & 0.41 & 0.09 & 1.87 & 0.25 \\
\hline \multicolumn{5}{|l|}{ Charlson score } \\
\hline (3) & 2.48 & 0.45 & 13.54 & 0.3 \\
\hline Syncope in last 7 days & 0.9 & 0.19 & 4.41 & 0.9 \\
\hline Altered mentation & 3.4 & 0.98 & 11.78 & 0.05 \\
\hline Lactic acid level, peak & 1.51 & 1.26 & 1.82 & $<0.0001$ \\
\hline
\end{tabular}

nal scores were: GBS AUC $=0.652(95 \% \mathrm{CI}$ : $0.557-0.747$, $\mathrm{P}=0.006)$, RS-PE AUC $=0.675$ (95\% CI: $0.586-0.765, \mathrm{P}=$ $0.001)$, RS-C AUC $=0.742$ (95\% CI: $0.633-0.850, \mathrm{P}=0.003)$. Venous LA was incorporated into each of these scoring modalities using the methods described above. The incorporation of LA improved the predictive value for inpatient mortality for GBS, RS-PE, and RS-C, although the increment was not statistically significant for L2-RS-C and L3-RS-C scores (where 2 and 3 points were added when LA level was $>2.75 \mathrm{mmol} / \mathrm{L}$, respectively). The highest statistically significant improvement in AUC was seen for RS-PE, especially when 3 extra points was assigned for $\mathrm{LA} \geq 2.75 \mathrm{mmol} / \mathrm{L}(0.675 \mathrm{vs} .0 .774$, $\mathrm{P}=0.012)$, which was comparable to or better than the RS-C $(0.742, \mathrm{P}=0.003$ ( $\mathrm{P}$ value not show in the Table $)$ ).

\section{Discussion}

In this retrospective study, we found that venous LA is an independent predictor of inpatient mortality in unselected patients presenting with acute gastrointestinal hemorrhage. Furthermore, the GBS, RS-PE, and RS-C scores, which were originally developed in the setting of upper GI hemorrhage are valid and applicable for the prediction of in-hospital mortality in unselected GIB patients. The AIMS65 score did not distinguish survivors from non-survivors. We also showed that incorporation of venous LA into GBS and RS showed statistically significant improvement in the power of the risk scores to discriminate inpatient mortality status. Importantly, the performance of the modified RS-PE (AUC 0.774 for L3-RS PE) was comparable to or better than that of the original RS-post endoscopic score (AUC 0.742), providing vital prognostication and risk-stratification for front-line providers before any diagnostic or therapeutic intervention has taken place.

The main strength of this study is that it aims to obviate a need to determine the site of GIB by demonstrating the validity of existing and modified clinical risk scores in mixed popula- tion of GIB patients. To our knowledge, this is the first study that has evaluated the utility of combining existing risk scores as well as the incremental value of venous LA in unselected GI bleed patients.

Our study is consistent with several other studies that evaluated the role of LA as an independent prognostic marker in patients with upper GI bleed. Stokbro et al [10] evaluated the incremental role of LA to clinical risk scores, specifically in upper GIB patients. They showed a statistically significant improvement in risk scores for predicting mortality by incorporating points for elevated arterial LA, at a cut-off value of $1.98 \mathrm{mmol} / \mathrm{L}$. However, the low AUC of the modified scores in their study tempered the enthusiasm for its clinical utility. This is due to the low AUC of the risk scores prior to LA incorporation ( 0.54 for GBS and 0.66 for RS-PE, compared to 0.652 and 0.742 , respectively, in our study). There are differences between our study and Stokbro et al that limit direct comparisons: 1) The patient population in our case is all GIB patients vs. upper GIB; 2) Our study evaluated only inpatient mortality vs. 30-day mortality; 3) We assessed venous LA vs. arterial LA, which may explain higher LA cut-off in our study since venous lactate tend to be slightly higher compared to arterial values [14]. Furthermore, in contrast to our finding, they found that the AIMS65 score was a superior predictor of inhospital mortality in contrast to our findings. Multiple studies have demonstrated that the AIMS65 scoring system may be the most accurate and useful predictor for all cause in-hospital and 30-day mortality [15-18]. Lee et al [9] evaluated patients presenting with non-variceal upper GI bleeding, and found that elevated levels of venous LA were associated with increased 30day mortality and higher rates of re-admission within 7 days. They incorporated LA into the AIMS65 score, and found that while the modified LA-AIMS65 score did have a higher AUC, the results did not reach statistical significance. It's important to highlight that the AIMS65 utilizes altered mental status as one of its criteria, a finding that is subject to inter-operator variability. In contrast, the RS-PE, RS-C, and GBS scores use 

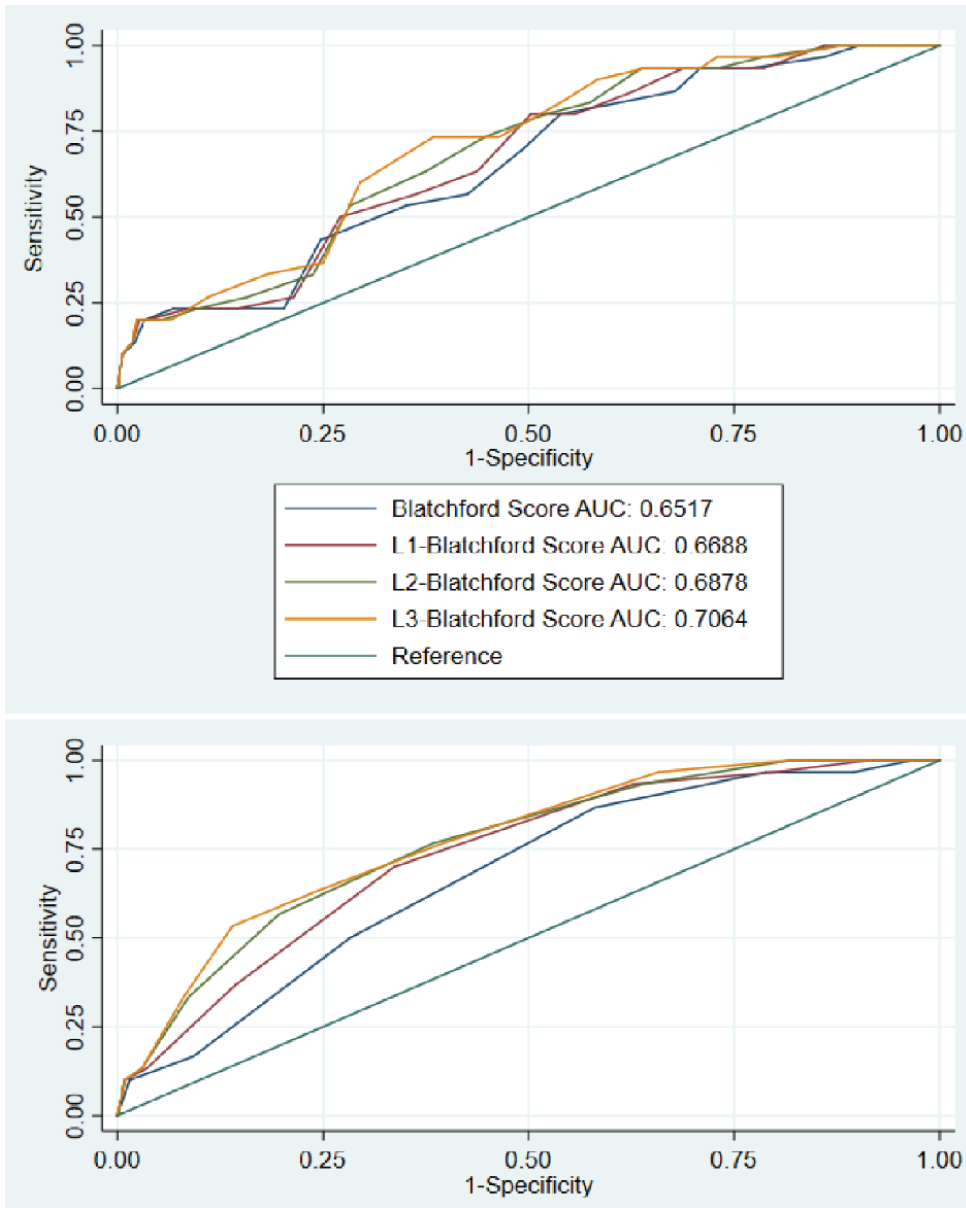

Rockall Pre-Endoscope Score AUC. 0.6754

L1-Rockall Pre-Endoscope Score AUC: 0.7332

L2-Rockall Pre-Endoscope Score AUC: 0.7624

L3-Rockall Pre-Endoscope Score AUC: 0.7741 Reference

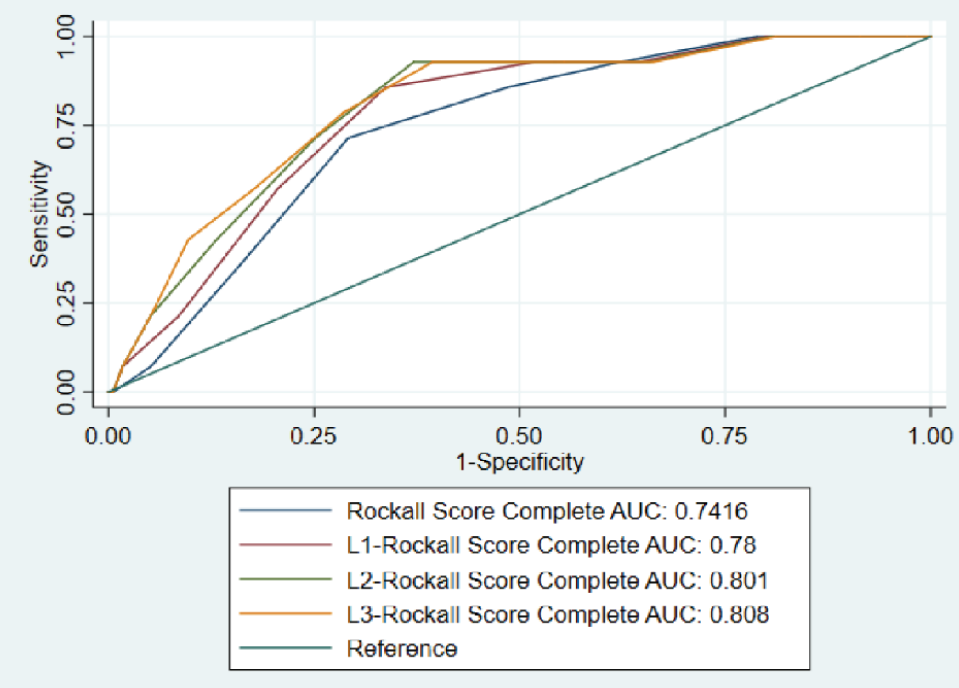

Figure 1. ROC curves comparing the original and modified Blatchford scores (top panel), Rockall pre-endoscope score (middle panel), and Rockall post endoscopic (complete) scores (bottom panel). 
Table 4. Comparison Between Original Clinical Risk Scoring Systems and Modified Scoring System Incorporating Points (1, 2, or 3) for Lactic Acid $\geq 2.75 \mathrm{mmol} / \mathrm{L}$

\begin{tabular}{|llllll}
\hline \multirow{2}{*}{ Score } & Area & P value & \multicolumn{3}{c}{ 95\% Confidence interval } \\
\cline { 5 - 6 } & & & Lower bound & Upper bound & P value \\
\hline Blatchford score & 0.652 & 0.006 & 0.557 & 0.747 & Blatchford score vs. \\
L1-Blatchford & 0.669 & 0.002 & 0.578 & 0.760 & 0.005 \\
L2-Blatchford & 0.688 & 0.001 & 0.600 & 0.775 & 0.003 \\
L3-Blatchford & 0.706 & 0.000 & 0.621 & 0.792 & Rockall pre-endoscopy vs. \\
Rockall Pre-Endoscopy & 0.675 & 0.001 & 0.586 & 0.765 & 0.003 \\
\hline L1-Rockall pre-endoscopy & 0.733 & 0.000 & 0.649 & 0.818 & 0.007 \\
L2-Rockall pre-endoscopy & 0.762 & 0.000 & 0.680 & 0.844 & 0.012 \\
L3-Rockall pre-endoscopy & 0.774 & 0.000 & 0.694 & 0.854 & Rockall score-complete vs. \\
Rockall Score-complete & 0.742 & 0.003 & 0.633 & 0.850 & 0.040 \\
L1-Rockall-complete & 0.780 & 0.000 & 0.677 & 0.883 & 0.078 \\
L2-Rockall-complete & 0.801 & 0.000 & 0.698 & 0.904 & 0.129 \\
\hline L3-Rockall-complete & 0.808 & 0.000 & 0.703 & 0.913 & \\
\hline
\end{tabular}

objective lab and vital sign-based criteria, which can decrease error associated with inter-operator variability.

Our study has several limitations that deserve commentary. The study was conducted in a single academic tertiary care referral center, and the design was retrospective. Nearly half of the patients had serum LA levels obtained at the index of the admission; as LA may have been obtained because these patients were perceived to be more acutely ill, thus posing a possible selection bias. Only half of those with LA measured underwent endoscopic examination, likely as most of the remaining half were not suspected to have upper GI source of bleed.

In conclusion, venous LA is an important prognostic marker for inpatient mortality in unselected patients presenting with acute GI bleeding and it can improve the performance of GBS and RS-PE scores in this setting. Further investigation with larger prospective trials can explore the prognostic ability of venous LA, which may provide earlier decision making among front-line providers regarding blood and volume resuscitation, appropriate level of care, and early intervention.

\section{Grant Support}

None.

\section{Conflict of Interest}

All authors have reported that they have no conflict of interest relevant to the content of this paper.

\section{References}

1. Ghassemi KA, Jensen DM. Lower GI bleeding: epidemiology and management. Curr Gastroenterol Rep.
2013;15(7):333.

2. El-Tawil AM. Trends on gastrointestinal bleeding and mortality: where are we standing? World J Gastroenterol. 2012;18(11):1154-1158.

3. Diamantopoulou G, Konstantakis C, Kottoromicronu A, Skroubis G, Theocharis G, Theopistos V, Triantos C, et al. Acute lower gastrointestinal bleeding: characteristics and clinical outcome of patients treated with an intensive protocol. Gastroenterology Res. 2017;10(6):352-358.

4. Liang PS, Saltzman JR. A national survey on the initial management of upper gastrointestinal bleeding. J Clin Gastroenterol. 2014;48(10):e93-98.

5. Laine L, Shah A. Randomized trial of urgent vs. elective colonoscopy in patients hospitalized with lower GI bleeding. Am J Gastroenterol. 2010;105(12):2636-2641; quiz 2642.

6. El-Kersh K, Chaddha U, Sinha RS, Saad M, Guardiola J, Cavallazzi R. Predictive role of admission lactate level in critically ill patients with acute upper gastrointestinal bleeding. J Emerg Med. 2015;49(3):318-325.

7. Ko BS, Kim WY, Ryoo SM, Ahn S, Sohn CH, Seo DW, Lee YS, et al. Predicting the occurrence of hypotension in stable patients with nonvariceal upper gastrointestinal bleeding: point-of-care lactate testing. Crit Care Med. 2015;43(11):2409-2415.

8. Kwon YH. Lactate parameters predict clinical outcomes in patients with nonvariceal upper gastrointestinal bleeding. J Korean Med Sci. 2017;32(11):1731.

9. Lee SH, Min YW, Bae J, Lee H, Min BH, Lee JH, Rhee PL, et al. Lactate parameters predict clinical outcomes in patients with nonvariceal upper gastrointestinal bleeding. J Korean Med Sci. 2017;32(11):1820-1827.

10. Stokbro LA, Schaffalitzky de Muckadell OB, Laursen SB. Arterial lactate does not predict outcome better than existing risk scores in upper gastrointestinal bleeding. Scand J Gastroenterol. 2018;53(5):586-591. 
11. Wada T, Hagiwara A, Uemura T, Yahagi N, Kimura A. Early lactate clearance for predicting active bleeding in critically ill patients with acute upper gastrointestinal bleeding: a retrospective study. Intern Emerg Med. 2016;11(5):737-743.

12. Shah A, Chisolm-Straker M, Alexander A, Rattu M, Dikdan S, Manini AF. Prognostic use of lactate to predict inpatient mortality in acute gastrointestinal hemorrhage. Am J Emerg Med. 2014;32(7):752-755.

13. Shrestha MP, Borgstrom M, Trowers EA. Elevated lactate level predicts intensive care unit admissions, endoscopies and transfusions in patients with acute gastrointestinal bleeding. Clin Exp Gastroenterol. 2018;11:185-192.

14. Kruse O, Grunnet N, Barfod C. Blood lactate as a predictor for in-hospital mortality in patients admitted acutely to hospital: a systematic review. Scand J Trauma Resusc Emerg Med. 2011;19:74.

15. Stokbro LA, Schaffalitzky de Muckadell OB, Laursen SB. Arterial lactate does not predict outcome better than existing risk scores in upper gastrointestinal bleeding. Scand J Gastroenterol. 2017;53(5):1-6.

16. Stanley AJ, Laine L, Dalton HR, Ngu JH, Schultz M, Abazi R, Zakko L, et al. Comparison of risk scoring systems for patients presenting with upper gastrointestinal bleeding: international multicentre prospective study. BMJ. 2017;356:i6432.

17. Martinez-Cara JG, Jimenez-Rosales R, Ubeda-Munoz M, de Hierro ML, de Teresa J, Redondo-Cerezo E. Comparison of AIMS65, Glasgow-Blatchford score, and Rockall score in a European series of patients with upper gastrointestinal bleeding: performance when predicting in-hospital and delayed mortality. United European Gastroenterol J. 2016;4(3):371-379.

18. Robertson M, Majumdar A, Boyapati R, Chung W, Worland T, Terbah R, Wei J, et al. Risk stratification in acute upper GI bleeding: comparison of the AIMS65 score with the Glasgow-Blatchford and Rockall scoring systems. Gastrointest Endosc. 2016;83(6):1151-1160. 